\title{
Interferometer-controlled scanning transmission X-ray microscopes at the Advanced Light Source
}

\author{
A. L. D. Kilcoyne, T. Tyliszczak, W. F. Steele, S. Fakra, P. Hitchcock, K. Franck, E. \\ Anderson, B. Harteneck, E. G. Rightor, G. E. Mitchell, A. P. Hitchcock, L. Yang, T. \\ Warwick and $\mathrm{H}$. Ade
}




\section{Interferometer-controlled scanning transmission X-ray microscopes at the Advanced Light Source}

\section{A. L. D. Kilcoyne, ${ }^{\text {a }}$ T. Tyliszczak, ${ }^{\text {b,c }}$ W. F. Steele, ${ }^{c}$ S. Fakra, ${ }^{c}$ P. Hitchcock, ${ }^{\text {a,b }}$ K. Franck, ${ }^{\mathrm{c}}$ E. Anderson, ${ }^{\mathrm{d}}$ B. Harteneck, ${ }^{d}$ E. G. Rightor, ${ }^{\mathrm{e}}$ G. E. Mitchell, ${ }^{\mathrm{e}}$ A. P. Hitchcock, ${ }^{\text {b }}$ L. Yang, ${ }^{\text {b }}$ T. Warwick ${ }^{\mathrm{c}}$ and H. Ade ${ }^{\mathrm{a} *}$}

${ }^{a}$ Department of Physics, North Carolina State University, Raleigh, NC 27895, USA, ${ }^{b}$ Brockhouse Institute for Material Research, McMaster University, Hamilton, ON L8S 4M1, Canada, ${ }^{\circ}$ Advanced Light Source, Lawrence Berkeley National Laboratory, Berkeley, California 94720, USA, ${ }^{d}$ Center for X-ray Optics, Lawrence Berkeley National Laboratory, Berkeley, California 94720, USA, and ${ }^{e}$ Dow Chemical, 1897 Bldg, Midland, MI 48667, USA. E-mail: harald_ade@ncsu.edu.

Two new soft X-ray scanning transmission microscopes located at the Advanced Light Source (ALS) have been designed, built and commissioned. Interferometer control implemented in both microscopes allows the precise measurement of the transverse position of the zone plate relative to the sample. Long-term positional stability and compensation for transverse displacement during translations of the zone plate have been achieved. The interferometer also provides low-distortion orthogonal $x, y$ imaging. Two different control systems have been developed: a digital control system using standard VXI components at beamline 7.0, and a custom feedback system based on PC AT boards at beamline 5.3.2. Both microscopes are diffraction limited with the resolution set by the quality of the zone plates. Periodic features with $30 \mathrm{~nm}$ half period can be resolved with a zone plate that has a $40 \mathrm{~nm}$ outermost zone width. One microscope is operating at an undulator beamline (7.0), while the other is operating at a novel dedicated bending-magnet beamline (5.3.2), which is designed specifically to illuminate the microscope. The undulator beamline provides count rates of the order of tens of $\mathrm{MHz}$ at highenergy resolution with photon energies of up to about $1000 \mathrm{eV}$. Although the brightness of a bending-magnet source is about four orders of magnitude smaller than that of an undulator source, photon statistics limited operation with intensities in excess of $3 \mathrm{MHz}$ has been achieved at high energy resolution and high spatial resolution. The design and performance of these microscopes are described.

Keywords: X-rays; zone plates; scanning microscopy; NEXAFS.

\section{Introduction}

Structure and phenomena on a length scale from nanometres to micrometres are the focus of much current scientific research. For example, heterogeneous composition and chemical properties on this scale are key to the behaviour of multiphasic polymeric materials, biomaterials and composites. Furthermore, environmental and biological processes often involve microscopically variable chemistry. There is therefore a growing need for detailed compositional and chemical analysis at high spatial resolution. Transmission X-ray microscopy in both scanning (STXM) and full-field variants (TXM) is becoming increasingly important on account of the relatively low radiation damage and compositional information that is provided by near-edge X-ray absorption fine-structure (NEXAFS) spectroscopy. Various implementations of zone-plate-based scanning transmission
X-ray microscopes (STXM) have been developed during the past two decades at several synchrotron radiation facilities (Kirz \& Rarback, 1985; Kirz et al., 1995; Warwick et al., 1998; McNulty et al., 1998; Kaulich et al., 1999). These instruments have been used to study many different types of samples including polymeric (Ade et al., 1992, 1995; Ade \& Hsiao, 1993; Ade \& Urquhart, 2002), geochemical (Botto et al., 1994; Cody et al., 1998), environmental (Plaschke et al., 2002), magnetic (Kim et al., 2001), extraterrestrial (Flynn et al., 1998; Keller et al., 2000) and biological (Kirz et al., 1995) samples. Most STXM studies use the information provided by the NEXAFS of elements with absorption edges in the soft X-ray energy range, particularly of carbon, nitrogen and oxygen (Stöhr, 1992). The ability of STXM to adapt to a wide range of sample environments, such as magnetic fields (Kim et al., 2001), variable temperature (Wang et al., 2000) and polymeric and environmental samples in aqueous media (Neuhäusler et al., 1999; Mitchell et al., 2002), is an important aspect of the technique, as is the relatively low amount of radiation damage caused by soft X-rays (Rightor et al., 1997; Coffey et al., 2002). In order to scan the photon energy for NEXAFS we need to use a synchrotron radiation facility as a tunable source for these instruments. In order to acquire useful information in a reasonable time, high photon flux in a small spot is required. During the past decade STXMs have been operated exclusively at high-brightness undulator sources (Kirz et al., 1995; Warwick et al., 1998). Since the number of straight sections at high-brightness synchrotron facilities is very limited, the use of undulator sources greatly limits the potential for growth of STXM technology. Furthermore, since the overall utility of NEXAFS microscopy to a wide variety of fields is now without question, the use of high-brightness bending magnets would offer increased access.

Two STXMs that significantly extend prior instrumental capabilities have been commissioned at the Advanced Light Source (ALS) in Berkeley. Their design and performance are described and discussed. One of these microscopes is the upgrade to the firstgeneration STXM at the ALS and is located on undulator beamline 7.0. In an important new development, the other new microscope has been installed on a dedicated bending-magnet beamline (5.3.2) in order to explore and, if adequate performance is found, exploit the utility of high-brightness bending magnets for STXM illumination. The 5.3.2 beamline itself was highly optimized for STXM use and was described in a companion paper (Warwick et al., 2002). This bendingmagnet beamline and the STXM implemented there have been shown to have excellent performance. The STXMs described here will be designated by their present beamline number at the Advanced Light Source and will be referred to as the 5.3.2 STXM and the 7.0 STXM, respectively. These new STXMs share many design features and both use laser interferometry to accurately position the sample relative to the X-ray optics. We will first discuss the basic operating principles of a STXM and describe the prior state-of-the-art technology to provide context. Subsequently, the design of the new ALS STXM instruments will be presented and their performance will be discussed with results from the 5.3.2 STXM as examples.

\subsection{Basic operating principle of a zone-plate-based STXM}

In a scanning X-ray microscope, a small spot of X-rays is raster scanned relative to the sample to create an image one pixel at a time while a suitable signal is monitored under computer control. The most common signal is the photon flux that is transmitted through the sample (STXM). In addition, measurements in the scanning instruments have also used front-face electron yield, luminescence yield and X-ray fluorescence yield. Because of challenges in scanning $\mathrm{X}$-ray optics, samples are typically scanned relative to a stationary 
X-ray beam in a STXM. Two-axis $x, y$ piezoelectric stages have generally been used for fine motion over a limited field of view. This $x, y$ piezo stage itself is mounted on top of $x, y, z$ stepping motor stages that provide motion in excess of many millimetres for largescale images or coarse positioning of dispersed small samples. The small X-ray spot is produced by a zone-plate lens. This is a variableline-spacing circular diffraction grating.

In order to suppress unwanted diffraction orders that would decrease the available signal-to-background ratio, the zone plate is fabricated with a central stop. A slightly smaller pinhole, the order selection aperture (OSA), is placed between the zone plate and the sample at about 0.75 times the zone-plate-to-sample distance. This allows only the positive first diffraction order to pass (see Fig. 1). Careful transverse and longitudinal alignment of the OSA with the zone plate is an essential aspect of tuning and operating a STXM. The alignment determines the imaging properties of the microscopes and the higher-order spectral contamination that may pass the zone-plate/ OSA arrangement.

The numerical aperture (NA) of a zone plate, i.e. the largest diffraction angle for the variable zones, is determined by the size of the outermost zone width $\Delta r$ at a fixed wavelength of the incident $\mathrm{X}$-rays $[\mathrm{NA}=\lambda /(2 \Delta r)]$. For fully coherent illumination, plane-waves or point sources, the Raleigh resolution of a zone plate, which corresponds to the first minimum of the Airy diffraction pattern, depends on the size of the central stop relative to the size of the zone plate and ranges from $0.9 \Delta r$ to $1.22 \Delta r$ (Michette, 1986). In practice, a finite source is used for illumination, and the diffraction pattern is convolved with the de-magnified finite source to obtain the actual point-spread function of the STXM. The illumination parameter $p=d \Theta / \lambda$, where $d$ is the illuminating source size and $\Theta$ is the full acceptance angle of the zone plate, is a convenient way to characterize the illumination. $p=0.5$ corresponds roughly to the halfAiry-disk illumination criterion, and represents a good trade-off between flux and achievable spatial resolution (Kirz et al., 1995). The point-spread function and the modulation-transfer function of a zone plate as a function of $p$ have been calculated (Winn et al., 2000), and the result shows in great detail how the imaging characteristics depend on the illumination characteristics. Random or systematic departures of the zones from circularity will also degrade the optical properties of a zone plate. In order to achieve adequate performance the zones have to be placed with an absolute accuracy of about $0.3 \Delta r$ (Michette, 1986). The achievable resolution depends on the capabilities of the zone-plate-fabrication technology and on the

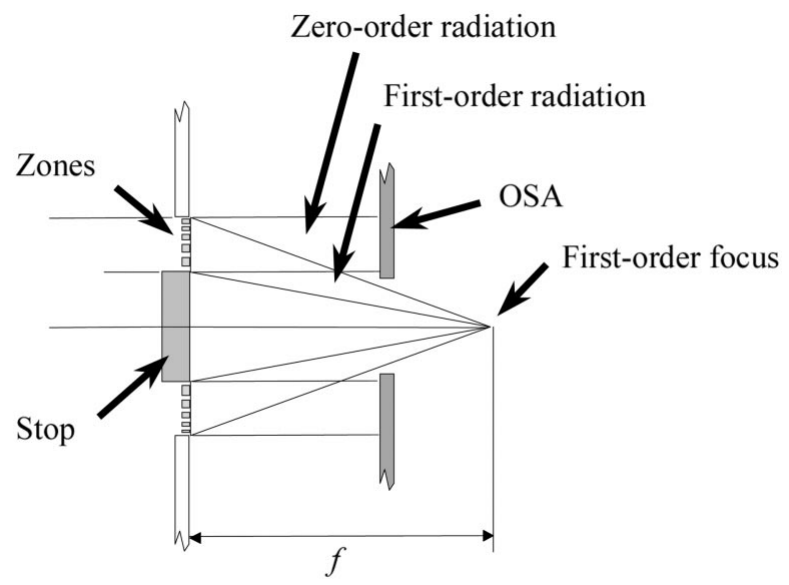

Figure 1

Focusing scheme of a STXM degree of coherence of the illuminating beam. Zone plates with outermost zone widths as small as $20-25 \mathrm{~nm}$ have been fabricated, and a spatial resolution sufficient to resolve half-period features 25-30 $\mathrm{nm}$ in size has been achieved (Anderson et al., 2000; Spector et al., 1997; Schneider et al., 1995; Denbeaux et al., 2001). Details of soft X-ray optics for STXM have been discussed in articles by Jacobsen $e t$ al. $(1991,1992)$.

Zone plates are achromatic lenses with a focal length proportional to the photon energy. Thus, during the acquisition of NEXAFS spectra or an image-sequence at many energy values (sometimes referred to as 'stacks'), a STXM microscope has to be refocused synchronously with the photon energy changes (Jacobsen et al., 2000). Depending on the focal length of the zone plate used, this requires motions over $150-200 \mu \mathrm{m}$ for a $30 \mathrm{eV}$-wide C $1 \mathrm{~s}$ NEXAFS scan or $>1 \mathrm{~mm}$ for a change between different absorption edges. This range can typically only be covered with conventional roller-bearing or crossed-roller-bearing stages. Unfortunately, these stages exhibit transverse motion, which is referred to as run-out, of the order of several hundreds of nanometres during a translation. Unless controlled, the run-out results in a relatively random misregistration of subsequent images of the same sample area or a 'blurring' of the spot size sampled during the acquisition of a spectrum from a point on the sample. If in-focus point spectra are acquired from small highly contrasting sample features, the spectra will contain artifacts with large spectral amplitudes if the photon beam moves on and off a feature of interest because of uncorrected transverse run-out.

Overall, a STXM requires control and operational adjustment of the $z$ position of the zone plate, at least $x$ and $y$ control of the OSA, $x$, $y$ and $z$ control over the sample, and generally $x, y$ and $z$ control over the detector.

For alignment, since the X-ray beam is fixed in space externally, either the zone plate or the whole instrument needs to be translated in $x$ and $y$ relative to the $\mathrm{X}$-ray beam with about $10 \mu \mathrm{m}$ precision. The translation stage that provides the focusing has to be aligned in two angles to be parallel with the externally defined optical axis. The rotational degree of freedom around the optical axis, i.e. roll, is not important.

\subsection{Prior state-of-the-art technology}

Pioneering efforts to construct the first soft X-ray STXM were made in the early 1980s (Rarback et al., 1984) at bending-magnet beamline $\mathrm{U} 15$ at the vacuum ultraviolet ring at the National Synchrotron Light Source (NSLS). The U15 STXM utilized a toroidal-grating monochromator with a resolving power $E / \Delta E=200$ at photon energies within the so-called water window between the absorption edges of oxygen and carbon. An initial spatial resolution of about $300 \mathrm{~nm}$ could be achieved with a zone plate that had an outermost zone width $\Delta r=150 \mathrm{~nm}$. Typical count rates were on the order of $10 \mathrm{kHz}$.

The Stony Brook effort was first relocated within the NSLS to a temporary undulator at beamline X17 in 1986-1987 (Rarback et al., 1988) and, subsequently, in 1988-1989 (Buckley et al., 1989), to its present location at beamline X1A. The spatial resolution improved to $75 \mathrm{~nm}$ in 1988 (25-75\% knife-edge test) with a $50 \mathrm{~nm}$ outermost zone-width zone plate (Rarback et al., 1988). By 1991, $35 \mathrm{~nm}$ halfperiod features could be resolved (Jacobsen et al., 1991; Kirz et al., 1992), and, by 1997, half-period features of less than $30 \mathrm{~nm}$ in size could be resolved (Spector et al., 1997). Typical count rates with the NSLS X1A microscope for operation near the carbon edge and with low higher-order spectral contamination are about $500 \mathrm{kHz}$. For 
much of the past two decades, the Stony Brook efforts have defined the state-of-the-art in STXM technology.

The X17 and the initial X1A STXM used a custom-built laser interferometer that included custom electronics to provide highspeed control and readout of the sample position relative to a reference prism (Rarback et al., 1988; Shu et al., 1988). These microscopes were designed to be used at a fixed photon energy within the water window. The interferometer had a least-count step size, or sensitivity, of $31.5 \mathrm{~nm}$ and was somewhat difficult to maintain. This sensitivity was insufficient for the high spatial resolution achieved, and, when integrated capacitance-controlled scanning stages with much higher sensitivity became available, from Queensgate and later from Physik Instrumente, two further incarnations of the Stony Brook STXMs were developed, in 1990-1992 and 1998-2000, respectively, which could take full advantage of the improvements made in zone-plate technology. Neither of these microscopes was designed to control run-out directly in hardware or firmware. Inaccurate registration between images is corrected with post processing based on image-correlation procedures (Jacobsen et al., 2000). Point spectra and line spectra cannot be measured at well defined locations with an accuracy near the spatial resolution limit. This information has to be extracted from image sequences after data acquisition. Details about the Stony Brook microscopes have been published (Jacobsen et al., 1991; Maser et al., 2000; Feser et al., 1998). The Stony Brook team also developed a cryo-STXM with tomography capabilities (Wang et al., 2000).

At the ALS, a STXM was developed in the mid-1990s by a team that included several of the present authors (Warwick et al., 1998). The STXM was located on beamline BL7.0, an undulator beamline that was conceived and optimized for spectroscopy. This firstgeneration ALS BL7.0 STXM was based on a capacitance-controlled piezoelectric stage from Queensgate Inc. as a fine stage. It used Newport PM-500 stages as sample coarse stages and for the zoneplate $z$ motion. The coarse and fine stages could be rotated together to provide in-focus scans over the full field of view when polar sample rotation with respect to the polarization vector of the photon beam was desired (Kim et al., 2001). The interface electronics were based on VXI crate/modules controlled by a Unix workstation. A second Unix workstation was dedicated for a graphical user interface (GUI). The GUI and low-level interface software were written in Labview. Spatial resolution of $60 \mathrm{~nm}$ FWHM was measured in a knife-edge test (Warwick et al., 1999). Routine operation used a slightly incoherent beam that resulted in a degraded spatial resolution on the order of $100 \mathrm{~nm}$ with improved noise characteristics. The microscope suffered from significant run-out of the zone-plate fine and coarse stages $(>500 \mathrm{~nm})$ and from magnified Abbé errors, the latter due to an offcentre sample mount. In addition, the STXM operation has to be shared with several other instruments on BL7.0 on a daily timeshared basis. One of the microscopes described below is the upgrade to this first-generation STXM at the ALS.

A STXM, built by a group from King's College, London (Kenney et al., 1989), was operated for a short time at the Daresbury U6 undulator beamline. The Göttingen X-ray microscopy group developed and operated for a short period a STXM located at a bending magnet of the BESSY-I facility. This group is now developing a new STXM and a new full-field transmission X-ray microscope (TXM), which are both located at the same undulator at BESSY-II (Guttmann et al., 2001). Scanning X-ray microscopes at higher photon energy have been implemented at the Advanced Photon Source (McNulty et al., 1998) and at the European Synchrotron Radiation Facility (Kaulich et al., 1999). Several scanning photoemission microscopes (SPEM) have been built at a number of facilities. These
SPEMs could be used, but are not optimized, for transmission experiments (Ade et al., 1990; Ko et al., 1995, 1998; Marsi et al., 1997; $\mathrm{Ng}$ et al., 1994; Shin \& Lee, 2001; Welnak et al., 1995).

\section{Instrument description: the 5.3.2 and 7.0 STXMs at the ALS}

\subsection{Technical goals and requirements}

The new ALS STXMs will be used almost exclusively for NEXAFS spectromicroscopy. This requires energy scanning and continuous refocusing. Prior experience showed that post-processing of data via image-correlation techniques does not always eliminate the run-out or drift that occur during data acquisition of image sequences, the result being residual spatial degradation (Hitchcock et al., 2003). Some correlation algorithms can be unreliable, and tedious manual image registration may be required. Our goal is to acquire data in a sequence of photon energy values in registry at the spatial-resolution limit of the microscope. Run-out has to be eliminated at the hardware level or automatically compensated for during data acquisition. In addition, in order to eliminate systematic image shifts as the focal length is changed, the microscope mechanical reference system has to be precisely colinear with the optical axis defined by the X-ray beam. Low run-out and component colinearity mean that high-quality spectroscopic information from a few specific locations in the sample is immediately availability to the instrument operator, and thus the efficiency and productivity of the instruments are improved.

Although the mechanical design of these two new microscopes at the ALS differs in some ways, they share the same basic concepts and components. The main new feature is the differential measurement of the relative position of the zone plate and the sample, which greatly improved the run-out of the new microscopes compared with that of the old 7.0 STXM. The 5.3.2 STXM is described here in detail. Some of the differences of the 7.0 STXM are pointed out.

\subsection{System components}

The following major system components have been incorporated: zone-plate $z$ stage; OSA $x, y$ stages; sample $x, y$ coarse stage; sample $x, y$ fine piezo stage; sample $z$ stage; detector $x, y, z$ stage; interferometer system; mounting platform; passive vibration isolation to the floor and vacuum/He enclosure. The arrangement and stacking of these stages is shown schematically in Fig. 2. Fig. 3 shows threedimensional CAD drawings of the 5.3.2 STXM. Annotated photographs of the instrument as implemented at beamline 5.3.2 are shown in Fig. 4.

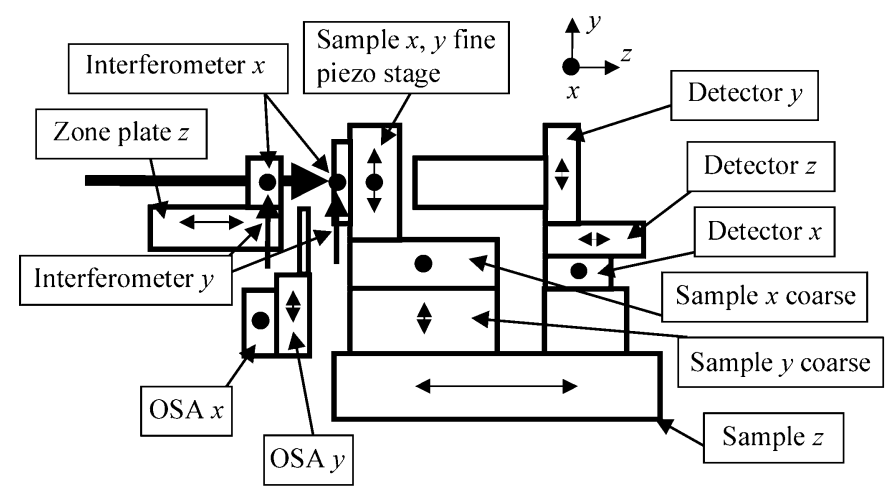

Figure 2

Schematic of the 5.3.2 STXM components. 


\subsection{Mounting platforms and vibration control}

Traditional air-table vibration-reduction systems are lowfrequency oscillators that isolate a heavy table from floor vibrations at frequencies above the lowest rigid-body mode, which typically resonates at about $1 \mathrm{~Hz}$. These systems are soft and can easily be moved by external forces. Such systems will not maintain the longterm angular alignment that is required with respect to the optical axis.

Rather than isolate the instruments from floor vibrations, we have built these microscopes on a rigid polymer composite base - referred to as polymer granite or polymer concrete (Zanite, Precision Polymer Casting) - that avoids resonant amplification at low frequencies. The

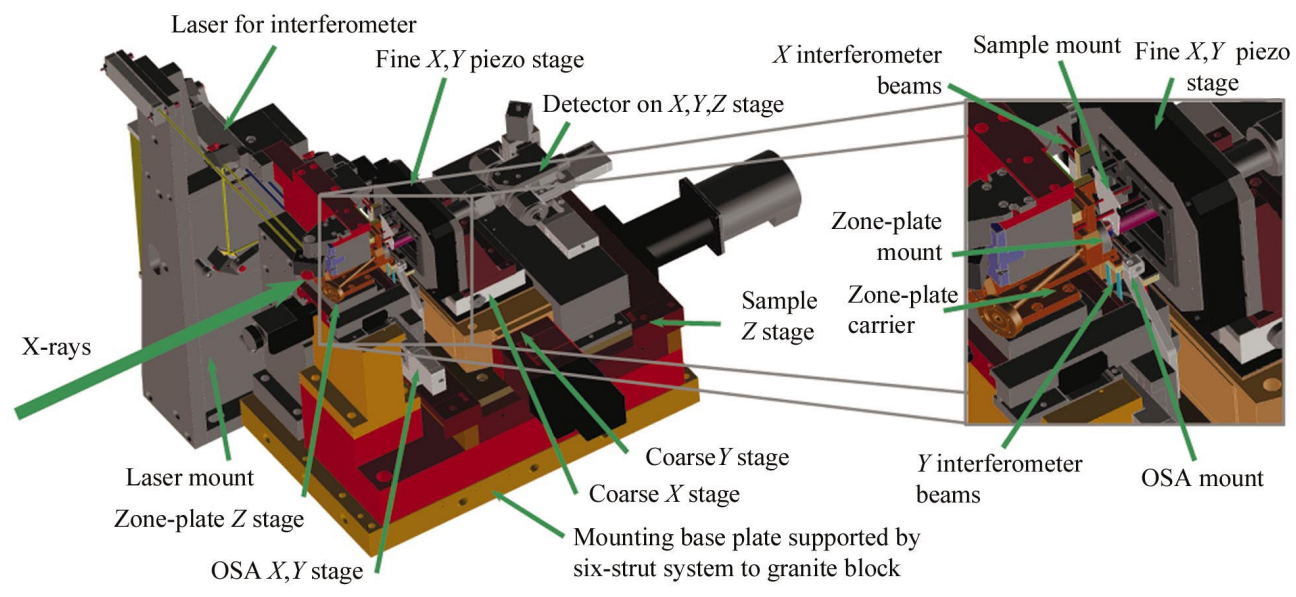

Figure 3

Annotated CAD drawings of the instrument at beamline 5.3.2.

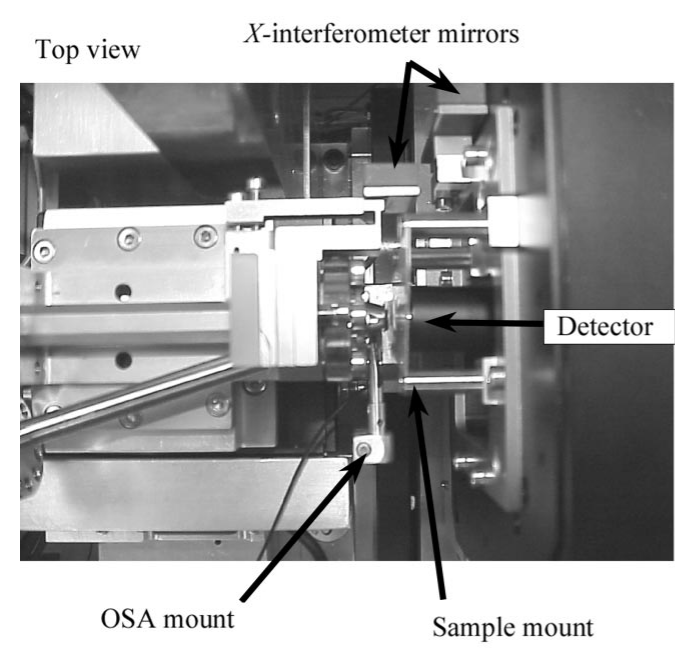

Figure 4

Photographs of the STXM at beamline 5.3.2. top surface of the polymer-granite block moves with the floor. The microscopes themselves are built so that only a small fraction of this amplitude (typically $10 \mathrm{~nm}$ ) shows up as problematic transverse vibration of the zone-plate lens relative to the sample. A multi-tier approach has been implemented. An inner mounting base plate for the STXM (see Fig. 3) is supported by a high-stiffness six-strut adjustable support system (Thur et al., 1997) mounted on the heavy polymer-granite block. This support system allows the adjustment of three translation and three angular degrees of freedom in order to accurately position the whole microscope and hence the zone plate and its translation axis with respect to the optical axis. The zone plate and sample are mounted from this 'inner' base plate with the set of stages as depicted in Figs. 2 and 3.

A vacuum vessel is employed, which covers the instrument without transmitting vacuum forces to the alignment mechanism and the aligned components inside and without transmitting vibrations of the beamline components to the instrument. The chamber seals to a metal plate embedded into the top of the granite, and this plate does not move or deflect in any appreciable way as the pressure is changed.

The ALS floor vibrations have a pronounced $110 \mathrm{~Hz}$ component. An attempt was thus made to keep resonance frequencies of all components above $130 \mathrm{~Hz}$. The polymer-granite block, which weighs about $1200 \mathrm{~kg}$, is supported on the floor by four 2 inch-thick polystyrene-foam pads. The foam has sufficient strength and long-term stability to support the massive block yet provides for some passive damping of vibrations. The 5.3.2 STXM six-strut support system uses 0.75 inch turnbuckle struts, with a stiffness of $52 \mathrm{~N} \mathrm{\mu m}^{-1}$ each, in a configuration that provides the most stiffness in the $y$ direction (three struts), a medium stiffness in the $x$ direction (two struts) and the least stiffness in the $z$ direction (one strut). This stiffness is matched to the relative magnitude of the horizontal $(x)$ and vertical $(y)$ vibrations in the floor, as well as the relative tolerance of the microscope to these vibrations: $x, y$ has a tolerance of $10 \mathrm{~nm}$ (one-quarter of the transverse resolution), while $z$ has a tolerance of about $100 \mathrm{~nm}$ (one-quarter of the longitudinal resolution). The three struts in the $y$ direction that support the $180 \mathrm{~kg}$ microscope result in a resonant frequency of about $150 \mathrm{~Hz}$. The BL7.0 STXM is lighter and uses 0.5 inch turnbuckle struts in a slightly different configuration. 


\subsection{Translation stages}

All translation stages of the 5.3.2 STXM have been selected for stiffness, range and accuracy, with much emphasis placed on stiffness. The sample $z$ stage is a large dovetail slide with a planetary rollerbearing spindle (Cleveland $\mathrm{GmbH}$ ). This slide is used to retract the scanner assembly and the pre-aligned detector for sample installation. The sample $z$ stage is also used to position the sample surface about $200 \mu \mathrm{m}$ downstream of the OSA. The sample $x$ and $y$ coarse scan stages were also chosen for high stiffness (UTM25 and UZM160, respectively, from Newport). The zone-plate $z$ stage is a small highprecision crossed-roller-bearing stage (M-126, Polytech PI) that is chosen for minimum run-out and for high stiffness with excellent vibration properties. This $z$ stage is moved or scanned as the photon energy is changed. The OSA $x$ and $y$ stages and the detector $x, y$ and $z$ stages are less critical (MFN series from Newport).

The sample $x, y$ piezo fine scan stage for the 5.3.2 microscope was chosen for its $200 \mu \mathrm{m}$ range and load capacity (P-527.3CL from Physik Instrumente). Even at the relatively high load of $500 \mathrm{~g}$, the resonance frequency as specified by the manufacturer is $190 \mathrm{~Hz}$, well above the resonance frequency of the stacked mechanical stages on which the piezo scanner is carried from the common base mounting plate. A smaller, lighter, $100 \mu \mathrm{m}$-range piezo stage (P-517.2CL from Physik Instrumente) was chosen for the BL7.0 STXM because of the higher speed requirements of that microscope.

Details of the geometrical arrangement in the 5.3.2 STXM of the zone plate, OSA, sample mounting and detector in relation to each other and to their translation stages are shown from three perspectives in Fig. 4. The optical path from the detector to the $\mathrm{Si}_{3} \mathrm{~N}_{4}$ exit window (not shown) of the UHV vacuum section, which is $0.5 \mathrm{~mm}$ upstream of the zone plate, is about $3.5 \mathrm{~mm}$.

\subsection{Interferometer}

Direct interferometric differential measurement of the position of the zone plate with respect to the sample is implemented in both STXMs, in order to meet the above-mentioned requirements for linearity, run-out and orthogonality. ${ }^{\mathbf{1}}$ Two Agilent Technologies 10719 A differential interferometers with 10889B PC Servo-Axis Boards (5.3.2), or with 10897B VME interferometer controllers (7.0), are used with the mirrors mounted to the sample and zone-plate carriers, respectively (see Fig. 3). The precision of this combination is $2.5 \mathrm{~nm}$ (least significant count). The orthogonality of the scan can be tuned by adjusting the sample mirror mounts. The peak-to-peak deviation from flatness of the mirrors is about $20 \mathrm{~nm}$. The laser interferometer is integrating over deviations from flatness within its $3 \mathrm{~mm}$-diameter beam, effectively averaging any small deviations from flatness over the much smaller image scan. Hence, once the mirrors are aligned, the transverse motion of the laser beam along the mirrors introduces neither image distortion nor appreciable run-out. The use of closed-loop piezo stage motion with a differential interferometer as a feedback is a major reason for the excellent performance of the 5.3.2 STXM.

After the axis of the zone-plate $z$-translation stage has been aligned to the optical axis by aligning the whole microscope via the struts, the reference mirrors on the zone-plate $z$ carrier can be tilted with adjustment screws such that the optical axis lies in a plane parallel to each mirror surface. In practice, the final angular adjust-

\footnotetext{
${ }^{\mathbf{1}}$ We considered using a monolithic stage for the refocusing translation with weak links that have virtually no run-out. Besides the difficulty of developing such stages with motion over 3 or even $5 \mathrm{~mm}$ as required, they would have involved a long mechanical coupling between the sample and the X-ray optics and their use would have most likely resulted in long-term drift.
}
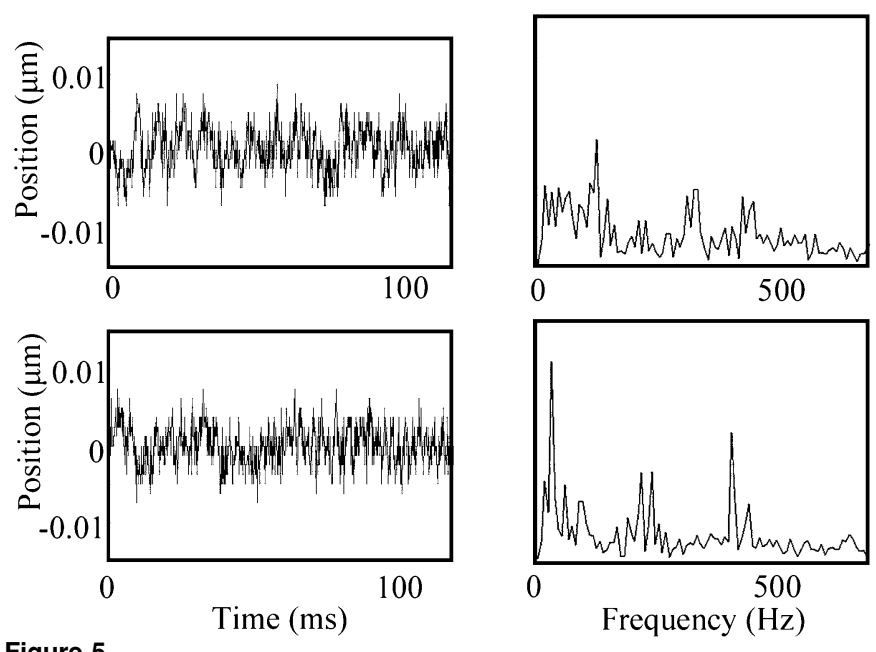

\section{Figure 5}

Ambient vibrations (left) and power spectrum (right) of the relative position of the zone plate and sample of the 5.3.2 STXM measured with the interferometer. Top: $x$ axis. Bottom: $y$ axis.

ment of these mirrors with respect to the optical axis is more readily accomplished with the six-strut support system. ${ }^{2}$

Other beneficial characteristics of the interferometer are linear and equal image magnification in the $x$ and $y$ directions with orthogonal $x, y$ axes. These magnification characteristics will aid the direct comparison of images from samples that have been rotated and hence the derivation of quantitative linear dichroic ratios. If such images from rotated samples cannot be made congruent, artifacts are introduced when calculating ratio or difference images.

The laser interferometer allows the direct measurement of the relative vibration of the zone plate and the sample. Ambient vibrations of the zone plate relative to the sample are seen with typical amplitudes of tens of $\mathrm{nm}$. When the interferometer is operated in a closed-loop mode, vibrations in both scan directions can be reduced to about $10 \mathrm{~nm}$ peak-to-peak. A typical measurement is displayed in Fig. 5. This measurement shows that the dominant modes have a few well defined frequencies. These results suggest that the performance of the microscopes should depend only on the quality of the zone plate and the illumination conditions provided by the beamline. These measurements also show that the 5.3.2 microscope, with closedloop control, is ready to use zone plates with spatial resolution better than $20 \mathrm{~nm}$.

The implementation of a laser interferometer also provides for a continuous absolute coordinate system with a field of view of over $20 \mathrm{~mm}$. This makes the operation and selection of the coarse and fine motion transparent to the operator. The operation is entirely under computer control. The large-field absolute coordinate system is also integral to a pre-indexing system based on visible-light microscopy. Areas of interest on a sample can be selected with a high-quality visible-light microscope, and the coordinates can be stored under computer control for later use. After sample transfer to an identical sample mount with a transfer accuracy of about $20-30 \mu \mathrm{m}$, the areas of interest can be recalled by the operator and easily found.

\subsection{Interface electronics and GUI for 5.3.2 STXM}

Fig. 6 is a simplified schematic of the 5.3 .2 control system. The differential interferometers ( $x$ and $y$ ) measure the transverse position

\footnotetext{
2 The resultant small misalignment of the $z$-translation stage results in a small displacement of the zone plate relative to the OSA as the energy is scanned.
} The effect is very small and can be readily tolerated. 
of the sample with respect to the zone-plate lens by means of mirrors attached to these components. The interferometers provide an accurate real-time measurement of the location of the X-ray spot on the sample. Digital signals from the interferometers are used to servo the piezo-scanning stage, in order to eliminate drift and vibrations under static conditions, and to servo the image coordinates during closed-loop scanning

The 10889B PC servo-controller cards were designed to control servo motors. These cards were found to work very well in our unusual application of using the 10889B analog control voltage for the piezo stage in closed-loop mode using the interferometer as feedback. This servo-controller provides full motion control with adjustable dead band, proportional-integral-differential (PID) and feed-forward parameters. The servo-loop on this board works with a selectable update frequency of up to $20 \mathrm{kHz}$. This high sampling frequency makes vibration reduction possible. The servo-loop inside the piezo controller is disabled since this loop uses capacitance position sensors inside the stage that cannot detect vibrations and drift of the structure or run-out of the $z$ stage. The interferometer/ piezo-stage servo-loop is opened when the sample is moved with the sample $x, y$ coarse stages. The loop is closed after the move in order to servo the stage to the new interferometer values. In this way an accurate and universal coordinate system is available over the entire field of view, which extends for tens of millimetres.

The typical scan mode records images one line at a time. The piezo stage is moved with a constant velocity; buffered acquisition and synchronization of the channel advance is used, and the velocity of the piezo stage is set so as to achieve the desired per-pixel dwell. During the scan of each line, the starting point is positioned a sufficient distance before the user-defined start-of-line to allow for stage acceleration. The servo-axis controller outputs a signal when the piezo stage is at the desired first-point position and thus starts the buffered and timed acquisition. While this method provides adequate precision at the current spatial resolution, we are developing an improved scheme that will provide on-the-fly use of the inter-

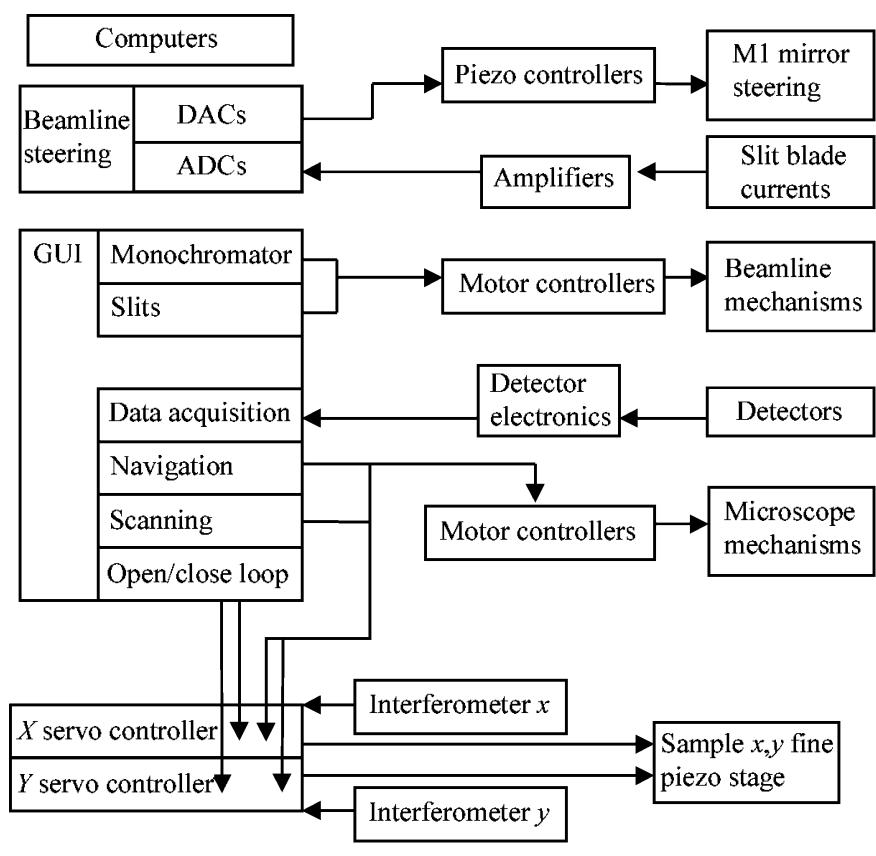

Figure 6

Scheme of the control system for STXM at beamline 5.3.2. ferometer signal to gate the acquisition at an accurate position at each pixel.

The system is capable of acquiring up to four counting channels, each 32 bit deep, and eight bipolar analog channels, with per-pixel dwells (time for all signals) as short as $100 \mu \mathrm{s}$. Owing to the time needed to decelerate, stop, return to start and re-accelerate the relatively massive piezo stage, the duty cycle for very fast acquisitions is much less than this point-to-point rate suggests. For a typical $10 \times 10 \mu \mathrm{m}$ scan, the time overhead for the fly-back and acceleration factors is about $200 \mathrm{~ms}$ per line. Thus, if the image is acquired with 200 pixels per line (i.e. a point spacing of $50 \mathrm{~nm}$ ), this is equivalent to a $1 \mathrm{~ms}$ per pixel overhead. Implementation of point-by-point on-thefly digital feedback will allow us to use bi-directional scanning, which will decrease the time not used for signal acquisition and improve the duty cycle.

The 5.3.2 STXM is operated through a single computer that controls all instrument and beamline parameters. The user interface controls 12 dimensions of motion of the various microscope stages (ZP, OSA, sample, detector), along with four aspects of the monochromator (grating angle, entrance slit, and horizontal and vertical exit-slit dimensions) and a fast-acting (1 ms open-close time) invacuum shutter. The latter is usually run in an automatic mode that closes the shutter at all times (including during the acceleration stage of each line in image-scan mode) except during data acquisition. Closing the shutter is essential to avoid unnecessary damage to radiation-sensitive samples. The computer is a dual-processor system running under the Windows 2000 operating system. Optimization of the number and contents of separate threads of the code is critical in order to achieve high performance without memory leakage and memory addressing collisions. A separate dedicated computer continuously optimizes the signals at the entrance and exit slits of the beamline via a feedback loop to two piezo transducers that control the angular fine adjustment of the toroidal M1 mirror of the beamline.

Ten translation stages are operated through two Newport universal controllers (ESP) that are computer interfaced with USB lines. The main sample $z$ stage is controlled by a conventional serial port. The pulse counting and analog acquisition is achieved through a National Instruments counter (PCI 6602) and multi-function I/O card (PCI MIO-16XE-10).

The control and acquisition software is designed to give the user full access to all motions of the instrument (microscope and beamline). The software has a flexible scan engine that allows single and multi-dimensional axis scanning using similar user interfaces. At the same time the GUI is structured so that only information necessary to the current task is provided to the user to avoid information overload. The front panel during image acquisition is shown in Fig. 7. Since spectromicroscopy involves a balanced use of the spectral and spatial domains, this panel gives equal weight to image and spectral presentation and manipulation. Novel aspects of the software include:

(i) Custom two-dimensional graph code that provides high-quality image presentation with updates of only the new information to avoid the overhead associated with full-screen rewrites.

(ii) Abstraction of the referencing and storage of the scan axes. The control aspects of all scan modes and associated data files are threedimensional, with $P, Q$ and $R$ axes assigned as needed to specific axes. Thus an image has $P=\{x\}, Q=\{y\}, R=E$, where $\{x\},\{y\}$ are the spatial coordinates of sample pixels (which can be defined arbitrarily) and $E$ is the photon energy. A focus scan has $P=\{x\}, Q=\{y\}$, $R=\{Z P-z\}$, where $\{x\},\{y\}$ are the spatial coordinates of a line across a contrast feature of the sample and $\{Z P-z\}$ is a sequence of 


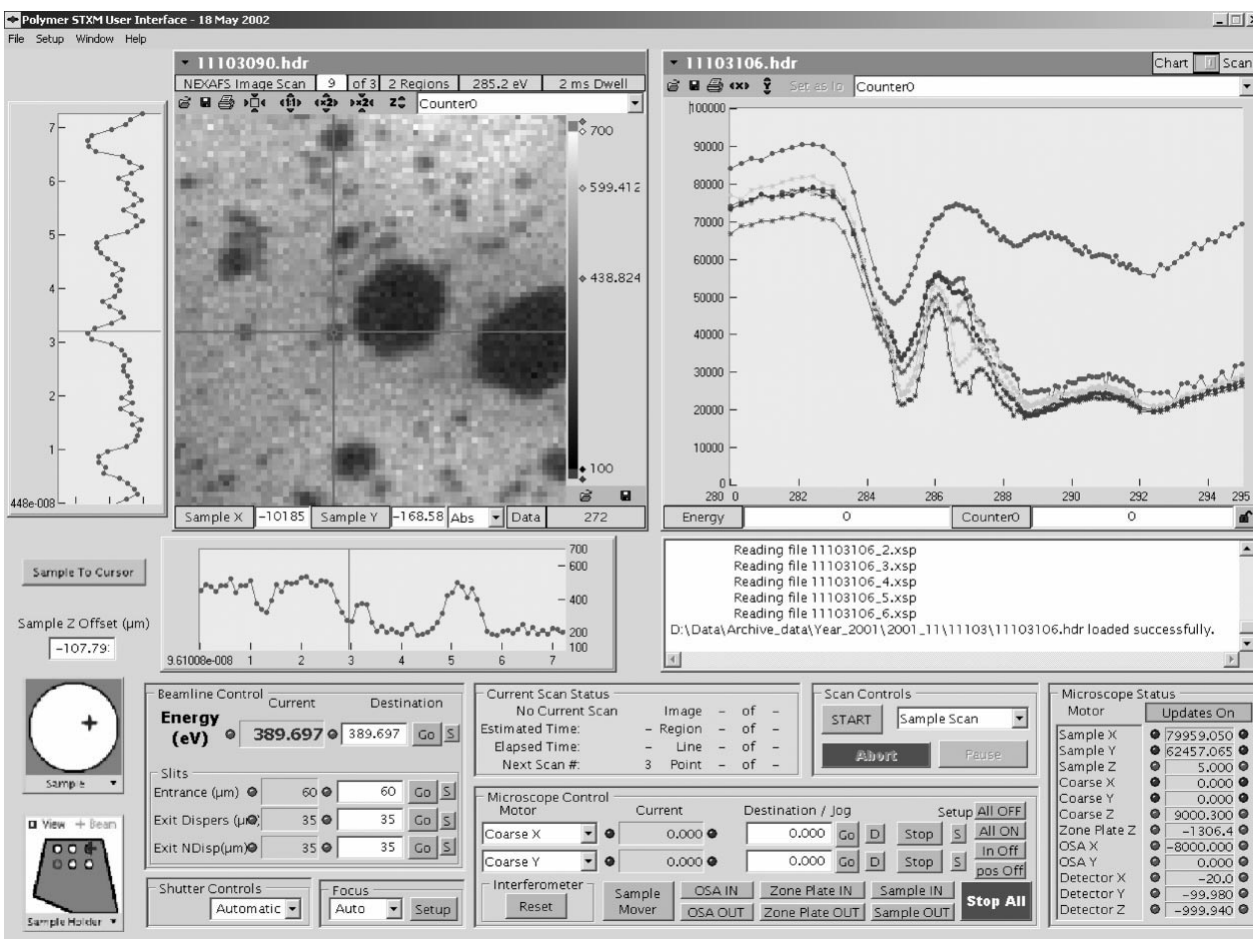

Figure 7

Graphical user interface for STXM at beamline 5.3.2.

zone-plate $z$ positions. As a third example, a NEXAFS image sequence has $P=\{x\}, Q=\{y\}, R=\{E\}$, where $\{x\},\{y\}$ are the spatial coordinates of sample pixels and $\{E\}$ is the set of photon energies that define a NEXAFS spectrum. Any scan mode can be executed over multiple regions in any of the scan domains (spatial or spectral), and the only limit on the number of data channels is that imposed by the hardware.

(iii) All data are written in ASCII in a novel self-defining file format that provides maximum compatibility with different computer systems and allows ready user access to instrument parameters. Each datum consists of a header file, which contains all microscope, beamline and scan parameters, and one or more associated files that contain the data. For example, a NEXAFS image scan that is acquired with the collection of four different signal channels (e.g. transmitted intensity, OSA monitor signal, total electron yield, luminescence) over three spatial regions and 100 photon energies would result in $4 \times 3 \times 100=1200$ image data files associated with a single header file. The naming convention for each one of these files specifies all aspects of region, data channel and image sequence number.

\subsection{Vacuum/He enclosure}

All of the microscope components are situated inside an aluminium chamber that can be evacuated to $10^{-6}$ torr or filled with helium. Compared with air, the $\mathrm{He}$ environment provides more photons on account of lower absorption and results in smaller interferometer drifts because of its lower index of refraction; furthermore the elimination of oxygen from the environment slows the degradation of polymeric materials during soft X-ray exposure (Coffey et al., 2002). At the same time, helium provides a thermally stable environment, once the fill reaches room temperature, because of the efficient cooling of the stepping motors and other electronic components inside the enclosure. All components, including piezo stage and stepping motors, are compatible with operation in a vacuum. This compatibility will allow further exploration of total-electron-yield detection methods in STXMs. All major adjustments and components of the microscopes are accessible via various removable panels on the chambers. The chambers have been constructed in such a way that a semi-automatic sample-transfer stage can be implemented in the future.

\subsection{Detectors}

Both microscopes employ phosphors to convert X-rays to visible light. The visible photons are counted by photomultiplier (PMT) systems with countrate capabilities in the tens of $\mathrm{MHz}$. The 5.3.2 detector is shown in Fig. 8. The front of the lucite pipe supports a very thin layer of a phosphor. Small single crystals of $\mathrm{CdWO}_{4}$ (CWO), $\mathrm{YAlO}_{3}$ : Ce (YAP) or $\mathrm{Gd}_{2} \mathrm{O}_{2} \mathrm{~S}: \operatorname{Pr}$ (GSO) are supported directly on a flat lucite tip. Granular phosphors (P11, P43 and P47) were deposited by sedimentation from a suspension. The granular-phosphor deposition process has to result in a layer that is thin enough to prevent light scattering, and hence loss of signal, yet sufficiently dense to cover the tip. The lucite tips are carefully shaped to increase the light collection of the granular phosphors by using refraction and critical internal reflection. The cone-shaped lucite increases the detection efficiency by more than $50 \%$ for the same sedimentation procedure. Different phosphors were compared by comparing the system efficiency with that of a silicon photodiode with a very thin oxide layer on the front surface. On average, the diode produces one electron-hole pair for each $3.6 \mathrm{eV}$ of X-ray energy deposited, and the X-rays are always absorbed in the silicon, except just above the oxygen edge $(530 \mathrm{eV})$. Thus, at a specific photon energy the absolute diode current gives the absolute photon flux. Table 1 shows a comparison of the efficiencies of the phosphors investigated. No systematic investigation of grain size and layer thickness was performed for the different granular phosphors. Rather, they were used as received and the sedimentation process

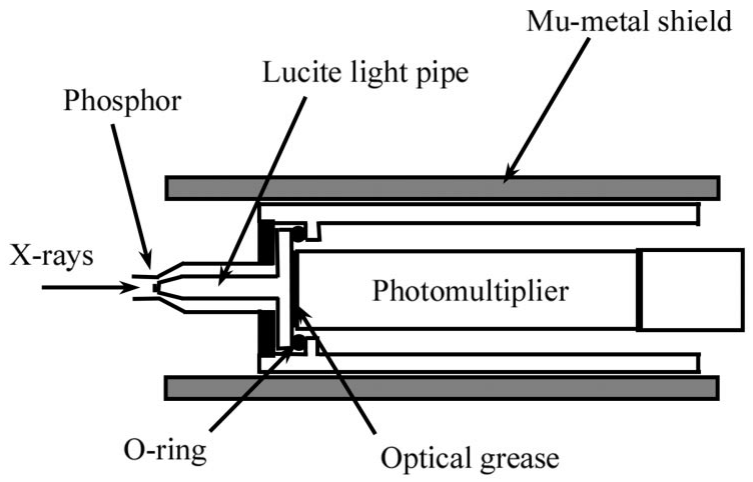

Figure 8

Geometry of the 5.3.2 detector for transmitted photons. 
Table 1

Efficiency of the PMT-based soft X-ray detector system with different phosphors at $390 \mathrm{eV}$.

Count rate with $60 \mu \mathrm{m}$ entrance, $35 \mu \mathrm{m}$ vertical exit and $35 \mu \mathrm{m}$ horizontal exit slits, scaled to $400 \mathrm{~mA}$ stored electron current. $\mathrm{CdWO}_{4}, \mathrm{YAlO}_{3}: \mathrm{Ce}$ and $\mathrm{Gd}_{2} \mathrm{O}_{2} \mathrm{~S}: \mathrm{Pr}$ single crystals are approximately $100 \mu \mathrm{m}$ thick.

\begin{tabular}{lll}
\hline Phosphor & Efficiency $(\%)$ & Count rate $(\mathrm{MHz})$ \\
\hline P43 flat & 27.3 & 14 \\
P43 cone & 41.0 & 21 \\
P11 flat & 12.8 & 6.56 \\
P47 flat & 6.9 & 3.54 \\
$\mathrm{CdWO}_{4}$ & 9.2 & 4.7 \\
$\mathrm{YAlO}_{3}: \mathrm{Ce}$ & 5.9 & 3 \\
$\mathrm{Gd}_{2} \mathrm{O}_{2} \mathrm{~S}: \mathrm{Pr}$ & 6.4 & 3.3 \\
\hline
\end{tabular}

was repeated for each phosphor until a uniform film was formed. The detector system may thus not be optimized yet, and our results reflect what can be achieved readily. P43 was chosen for both microscopes. It has no appreciable long-lived emission, which would otherwise cause streaking in images.

The detector system efficiency versus photon energy of a detector with P43 phosphor is shown in Fig. 9. The dependence of the efficiency on photon energy varies somewhat between deposited films. Fig. 10 shows the count-rate capability of a $\mathrm{P} 43$ detector. The detector system is linear up to about $5 \mathrm{MHz}$ of detected photons and only marginally nonlinear below $10 \mathrm{MHz}$. At higher photon rates the deadtime of the counting electronics becomes significant. The linear range of these detectors is sufficient for the count rates at the bending-magnet beamline 5.3.2. Nonlinearity, however, can be an issue for the higher count rates that are possible with an undulator beamline.

The mu-metal shielding of the PMT is extended towards the sample and together with the sample mount serves as an effective visible-light baffle against scattered laser light from the interferometer. The typical dark count rate is less than $200 \mathrm{~Hz}$. Compared with the signal, this value is very small and does not cause any spectral distortions, even for samples that are several optical densities thick.

\subsection{Suppression of higher-order spectral contamination}

The spectroscopic performance of the microscopes is degraded by the presence of higher-order photons - those at multiples of the

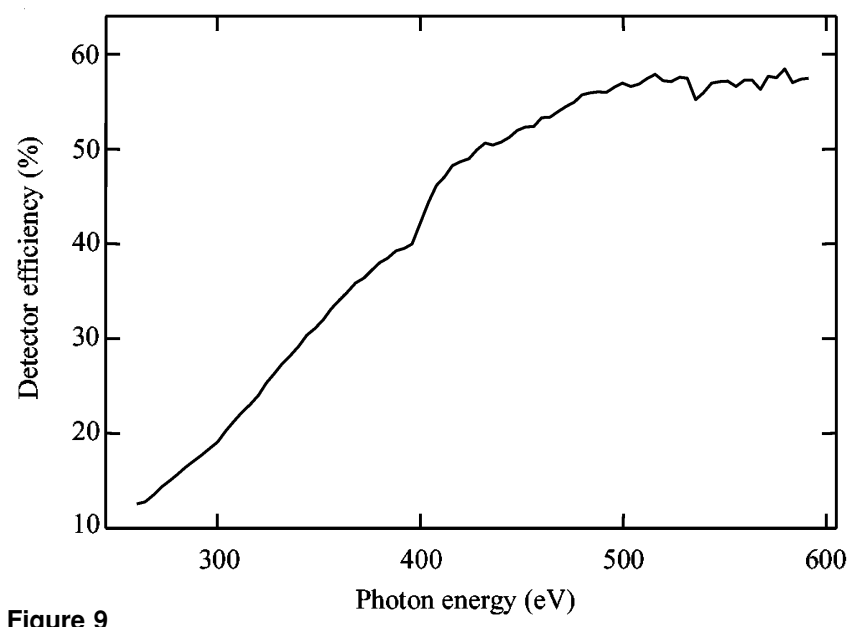

Efficiency of 5.3.2 P43/PMT counting system versus photon energy. Some variation occurs from one coating to another. selected photon energy. Typical soft X-ray monochromator diffraction gratings produce second- and third-order diffraction of photons with twice and three times the desired energy with $2-10 \%$ of the efficiency of the first order. A bending-magnet STXM is potentially rather sensitive to higher-order spectral contamination because of the intrinsically higher flux of the bending magnet at higher energies in the energy range of interest, the higher efficiency of the detector with photons with higher energy, and the increased penetration of higherenergy photons through the thin $\mathrm{Si}_{3} \mathrm{~N}_{4}$ membranes used to support the zone plate and for the exit window. Fortunately, an amplitude zone plate with a 1:1 mark-to-space ratio has a second-order diffraction efficiency close to zero. In reality, there is always some higher diffraction order intensity, but it can be very small. With proper positioning of the zone plate and the OSA, this results in a relatively effective suppression of second-order spectral contamination. Furthermore, the grating groove depth at beamline 5.3.2 was chosen to optimize the diffraction efficiency for $320 \mathrm{eV}$ photons, thus depressing higher-order spectral contamination over much of the important 280-420 eV energy range. For experiments near the carbon $K$-edge, any residual second-order light can be effectively and conveniently suppressed with a nitrogen-gas filter (Winesett \& Ade, 1997; originally only an oxygen filter was explored - it was later realized that nitrogen is a superior filter for work near the carbon edge). Second-order suppression by a factor of 10 has been achieved with a modest reduction in flux of about $50 \%$. Reduced reflectivity of grazing-incidence optics in the beamline at high photon energy reduces third-order spectral contamination. For working energies between 250 and $660 \mathrm{eV}$, third-order light can be attenuated particularly well by nickel coatings on the optics. This approach works well at beamline 5.3.2, which is not intended for use at energies above $600 \mathrm{eV}$.

\section{Results}

\subsection{Spatial resolution}

The 5.3.2 beamline follows design principles developed at beamline $\mathrm{X} 1 \mathrm{~A}$ at the NSLS (Winn et al., 2000) and provides very flexible illumination (Warwick et al., 2002). Because of the large phase space available at a bending magnet, independent control over a relatively large range of the zone-plate illumination parameter $(p)$ and resolving power $(E / \Delta E)$ has been achieved. For typical operating conditions the resolving powers at the carbon, nitrogen and oxygen edges

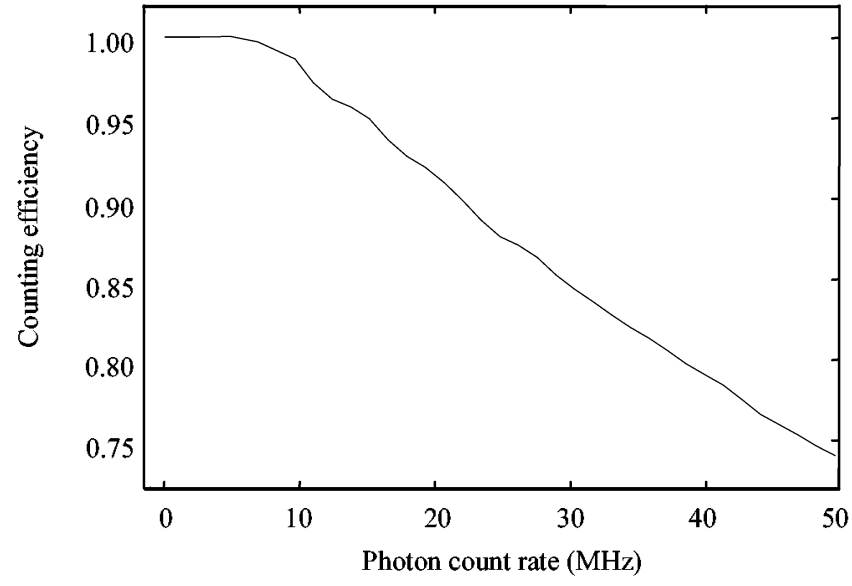

Figure 10

Linearity and non-linearity of the 5.3.2 P43/PMT detector system at high count rates. The fast pre-amp was divided by 10 
are in excess of 2000 but can be improved to greater than 5000 (Warwick et al., 2002). For high-spatial-resolution imaging, an illumination parameter (Winn et al., 2000) of between $p=0.5$ and $p=1$ is selected, which corresponds (at $310 \mathrm{eV}$ ) to exit slits of 10 and $20 \mu \mathrm{m}$ and to half-Airy-disk and full-Airy-disk illumination criterion, respectively. Various test patterns fabricated in $\mathrm{Au}$ with electronbeam lithography techniques and supported on $\mathrm{Si}_{3} \mathrm{~N}_{4}$ membranes have been imaged with a $180 \mu \mathrm{m}$-diameter zone plate with an outermost zone width of $40 \mathrm{~nm}$ and a central stop of $80 \mu \mathrm{m}$. Results from periodic features with a 1:1 line-to-space ratio are displayed in Fig. 11. Features as small as $30 \mathrm{~nm}$ half period can be resolved. The contrast for $40 \mathrm{~nm}$ and $30 \mathrm{~nm}$ features is $24 \%$ and $11 \%$, respectively. These results are as good as can be expected with this zone plate. The microscope is limited neither by mechanical vibrations nor by the use of the laser interferometer. The total overhead time of the image scan is about $1 \mathrm{~ms} \mathrm{pixel}^{-1}$, which is similar to the dwell time used for navigation (typically $<1 \mathrm{~ms}$ pixel $^{-1}$ ) but is small compared with that used for high-quality images (typically $3-5 \mathrm{~ms} \mathrm{pixel}^{-1}$ ) or spectra (typically $30 \mathrm{~ms}$ point ${ }^{-1}$ ). No astigmatism could be detected. Both horizontal and vertical features on the elbow test pattern displayed in Fig. 11 were in focus at the same $z$ location. Opening the vertical and horizontal exit slits greatly increases the intensity, at some cost in spatial and spectral resolution. An extreme case is $p=4$ and $E / \Delta E=1500$, for which count rates in excess of $20 \mathrm{MHz}$ could be achieved at the carbon $K$-edge while features $120 \mathrm{~nm}$ in size could still be resolved. All images, including those in Figs. 11 and 12, exhibit orthogonal linear magnification.

For quantitative NEXAFS analysis it is important that there be negligible unfocused light. Images of small (10 $\mu \mathrm{m}$ diameter $)$ pinholes have been acquired in order to verify the effective blocking of the zone-plate zero order and the absence of higher-order light from the beamline at higher energy. The contrast in these images from bright (pinhole) to dark (periphery of pinhole) is shown in Fig. 12. The integrated intensity of unfocused light at beamline 5.3.2 is typically less than $0.5 \%$ of the intensity of the zone-plate first-order focal spot.

\subsection{Image sequences, point and line spectra}

The interferometer servo-control of run-out works in both the $x$ and the $y$ direction. Image sequences ('stacks') register, one to
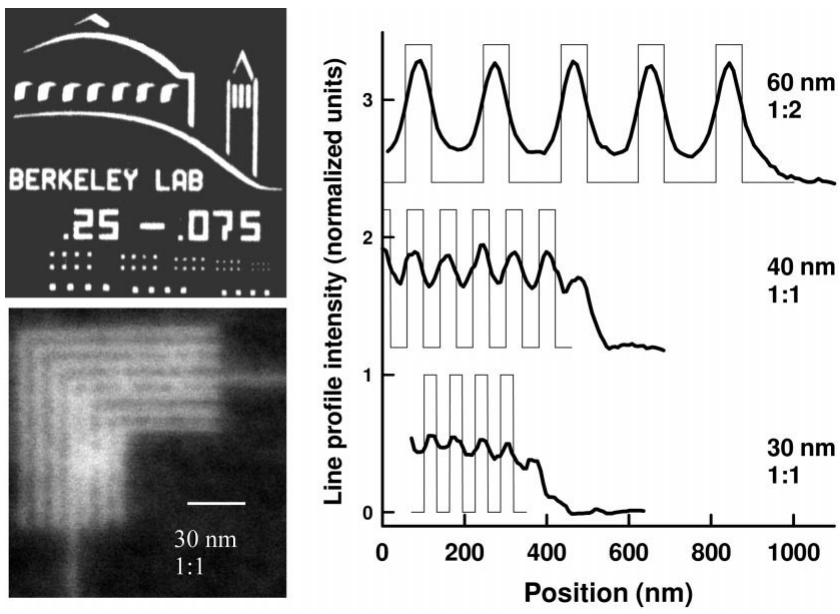

Figure 11

Images of test patterns and their contrast. Acquired with STXM at beamline 5.3.2. Top left: Berkeley logo and sequence of dots whose sizes range from $250 \mathrm{~nm}$ down to $75 \mathrm{~nm}$. Bottom left: $30 \mathrm{~nm}$ angular features with 1:1 mark:space ratio. Right side: line profiles of periodic structures with feature size and mark:space ratio as indicated. another, with very little spatial jitter. In order to demonstrate the precision of the registration, a sequence of images of a high-contrast test object (the $10 \mu \mathrm{m}$-diameter pinhole) was analysed to extract quantitative shifts. This test 'stack' is a sequence of 31 images taken with varying photon energy from 360 to $390 \mathrm{eV}$, with the zone plate translating longitudinally over a total distance of $175 \mu \mathrm{m}$ to stay in focus. A range of $175 \mu \mathrm{m}$ is sufficient for a NEXAFS scan at the carbon, nitrogen, and oxygen edges. Fig. 13 shows the residual alignment shifts in $x$ and $y$ from image to image of the sequence. These values were derived by tracking shifts in the peak of the twodimensional Fourier transform of images of an Au test pattern (Jacobsen et al., 2000). High-frequency run-out is controlled to about $20 \mathrm{~nm}$. Residual, still to be corrected, misalignment in the $y$ direction amounts to less than $40 \mathrm{~nm}$. If the interferometer is properly aligned, it will be possible to acquire images sequences, line scans and point spectra without degradation in spatial resolution. The long-term stability of this interferometer alignment in response to heavy use by many different researchers is still being assessed.

With demonstrably small transverse shifts as the zone-plate moves, NEXAFS spectra from small areas near the resolution limit can be extracted from image sequences without post-acquisition re-registration. Spectra can also be acquired in 'point' or 'line' mode. In 'point' mode the transmitted flux is measured with the microscope pointed to specific spatial features at each energy. In 'line' mode a line is scanned in the $x y$ plane and the transmitted intensity is measured on the line at each value of photon energy. The 'line' mode is useful if the sample has a transition from one chemical state in one region to a different state in another region. Fig. 14 shows spectra acquired from
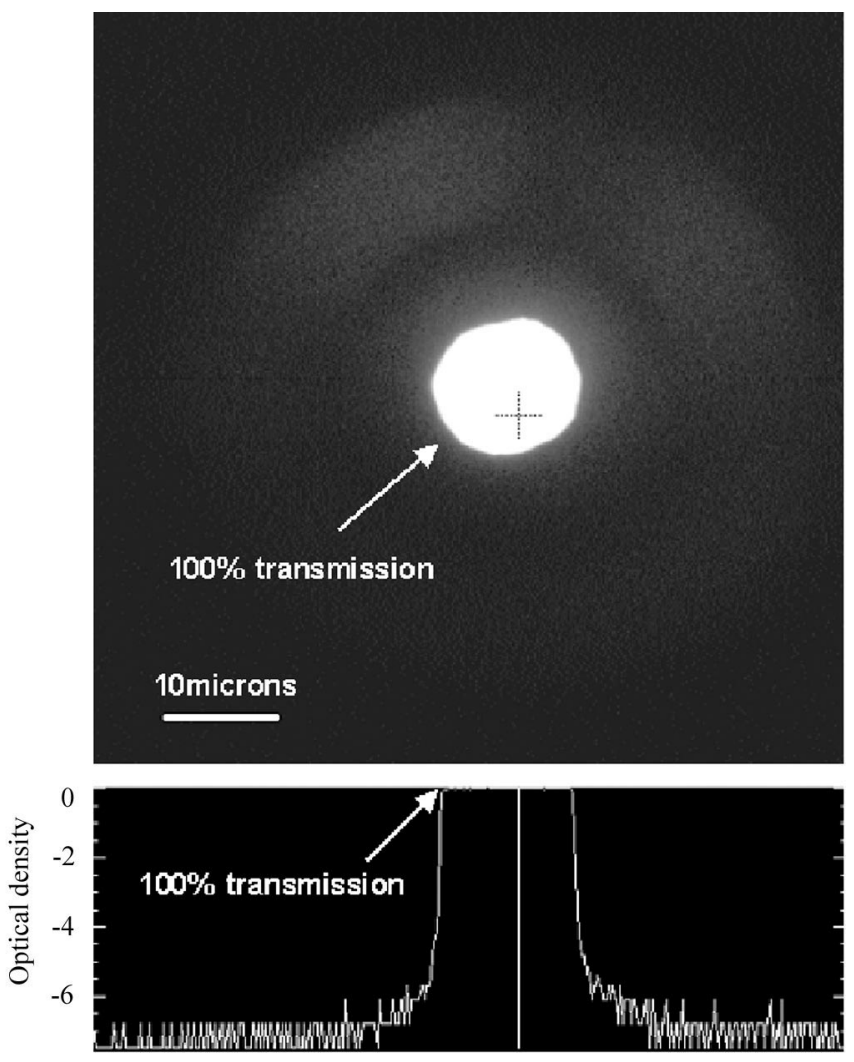

Figure 12

STXM image of a $10 \mu \mathrm{m}$-diameter pinhole acquired at $390 \mathrm{eV}$ showing negligible halo. (Image displayed on optical density scale. Line scan from location indicated by cross-hair in image.) 


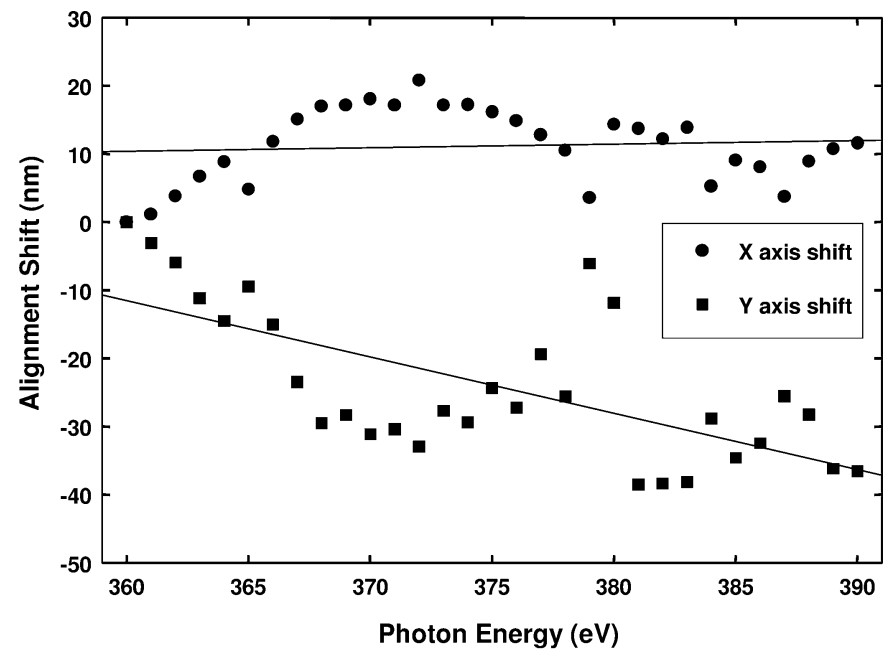

Figure 13

Residual alignment shifts in a servo-controlled image sequence with the 5.3.2 STXM. The shifts are extracted from correlation analysis of the image sequence (see text)
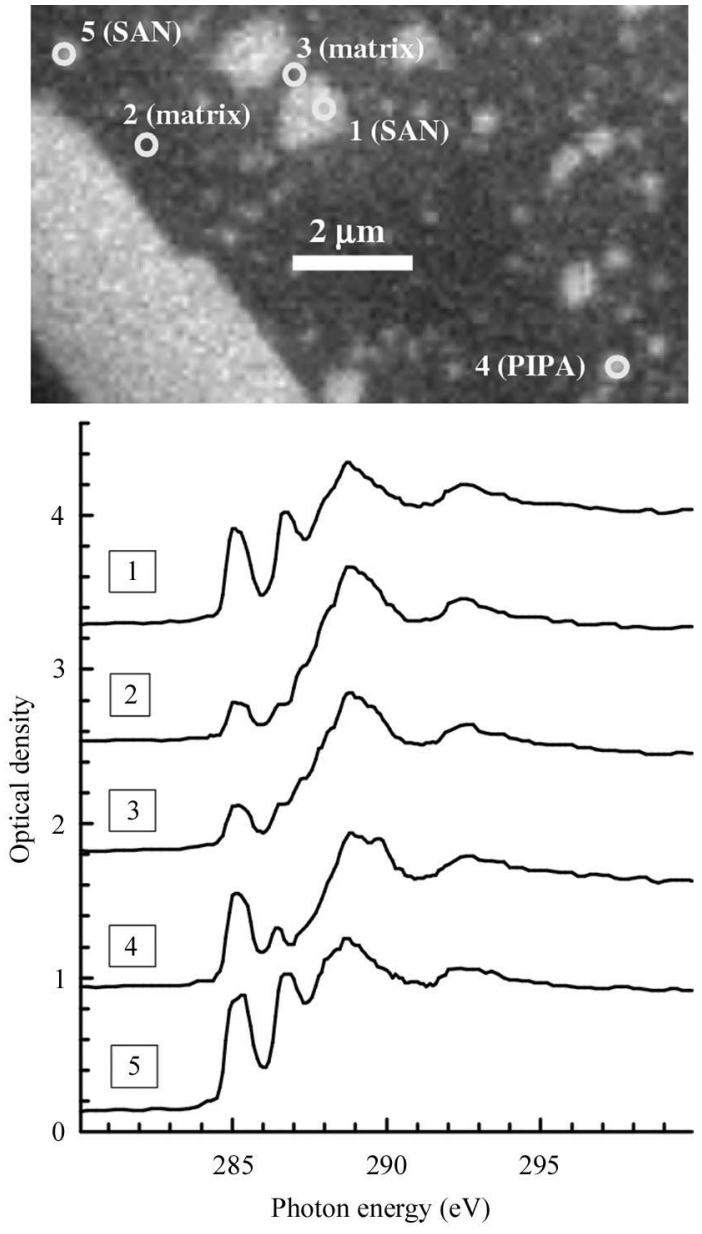

Figure 14

NEXAFS spectra extracted from specific points in the sample. Navigation to these points at each value of the photon energy is under interferometer servo control. Two organic polymer species (SAN, PIPA) can be identified to particle sizes as small as about $200 \mathrm{~nm}$. a point on a polyurethane that contains styrene- $r$-acrylonitrile (SAN) and polyisocyanate polyaddition (PIPA) filler particles. The three chemical constituents - polyurethane matrix, SAN and PIPA - can be differentiated by their NEXAFS spectra. Spectra are measured cleanly down to the smallest particles, which are about $200 \mathrm{~nm}$. In addition to eliminating problems of drifting of point positions that plagued previous STXMs, the results plotted in Fig. 14 demonstrate the ability of the interferometrically controlled system to address desired features to better than $200 \mathrm{~nm}$ spatial precision.

Line-mode results are displayed in Fig. 15. The transmitted signal is recorded while a line trajectory is scanned across the sample at each value of the photon energy through the NEXAFS spectrum. Optical density is derived from the transmitted intensity and plotted versus photon energy ( $x$ axis) and distance along the line ( $y$ axis). Thanks to interferometer control the line across the sample is scanned at precisely the same trajectory and thus the spectra of chemically distinct regions along the line are seen as horizontal stripes in the 'line' spectral image (Fig. 15).
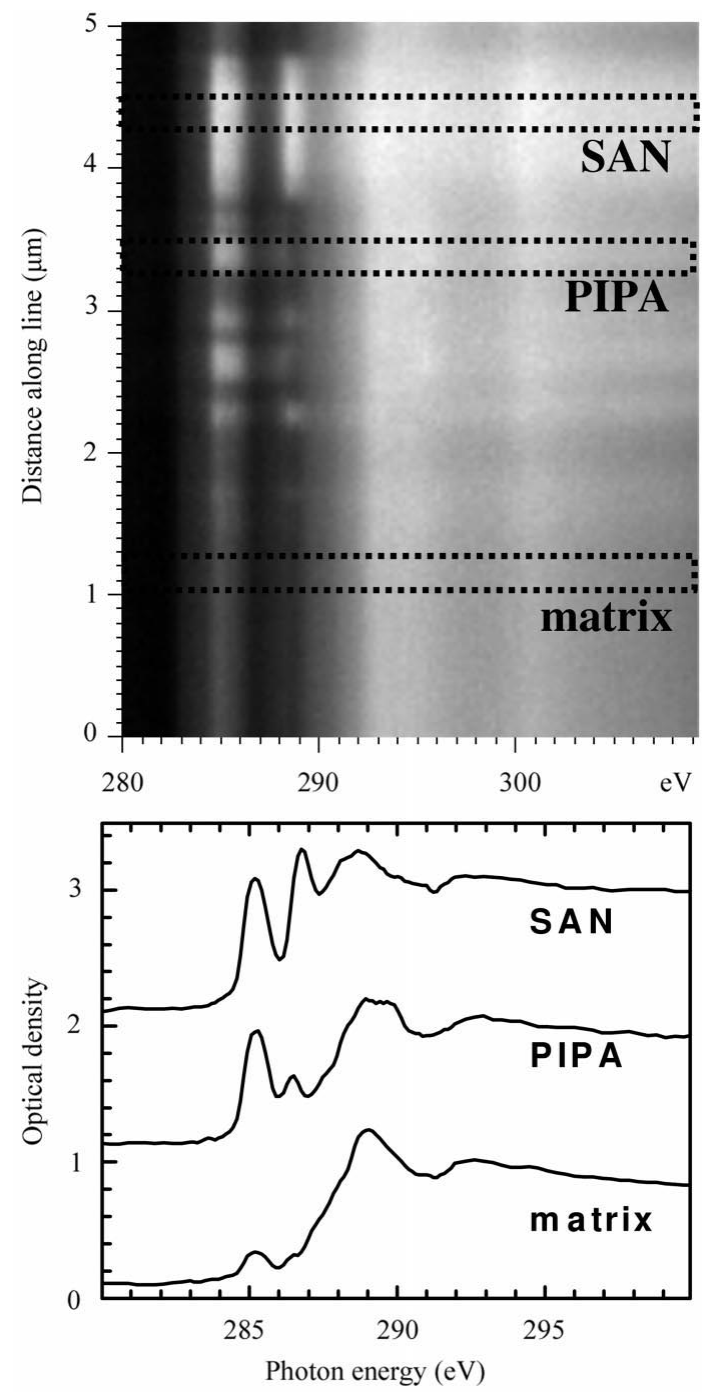

Figure 15

Line spectra obtained along a line in the sample, scanned under servo control at each value of the photon energy. The two-dimensional plot shows the optical density measured along the line versus the photon energy. Spectra are horizontal cuts across the two-dimensional plot. 


\section{Conclusion}

The laser interferometer-controlled instruments described here represent a qualitatively new design that has improved the capabilities of soft X-ray STXM technology and, in particular, the facilities at the ALS. The approach that we present provides for highly efficient data acquisition and minimizes data analysis and post-processing. Avoiding the use of active vibration isolation provides stable longterm positional and angular alignment with the X-ray beam. Smallamplitude vibrations of the zone plate with respect to the sample have been achieved. We observe only $10 \mathrm{~nm}$ peak-to-peak vibrations when the interferometer servo-loop is active. This result shows that the hardware of these microscopes is ready to take advantage of the zone-plate improvements that are anticipated in the near future and maybe even over the next decade.

The absolute accurate coordinate system based on the laser interferometer has made the distinction between coarse and fine motion transparent to the experimenter without introducing artifacts. Accommodation of accurate visible-light pre-indexing of the samples further enhances the efficiency of the 5.3.2 microscope.

The successful operation of a STXM at bending-magnet beamline 5.3.2 has significant implications. The ability to operate a STXM successfully at a bending-magnet beamline significantly lowers the overall costs for these instruments. In addition, the 5.3.2 STXM is the only STXM with dedicated illumination, so that it is available for STXM experiments whenever electrons are stored in the ALS ring. The gas filter for higher-order spectral suppression at 5.3.2 can be easily tuned by controlling the pressure; the retuning has no impact on the alignment or performance of the optical system.

A new dedicated beamline, BL11.0, which uses an elliptically polarized undulator as its source, is under construction. This beamline will add significant new capabilities by allowing control over the polarization (rotating linear as well as left/right circular). The 7.0 STXM is scheduled to move to BL11.0 in the fall of 2002.

We are grateful for the invaluable contributions made by Tim Harvell and Frank Milkowski from the NCSU machine shop, and Frank Zucca, John Pepper and Steve Klingler from the ALS vacuum group. Discussion with C. Jacobsen, J. Kirz, H. Padmore and C. Zimba contributed to developing the conceptual design. We thank $\mathrm{M}$. Howells for discussions about the possibilities and limitations of monolithic stages with low run-out; I. Koprinarov for help with the selection of the visible-light indexing microscope; and C. Jacobsen and J. Kirz for commenting on the manuscript. This work was supported by NSERC, the Canada Research Chair program, Dow Chemical, DOE (DE-FG02-98ER45737) and NSF (DMR-9975694).

\section{References}

Ade, H. \& Hsiao, B. (1993). Science, 262, 1427-1429.

Ade, H., Kirz, J., Hulbert, S. L., Johnson, E. D., Anderson, E. \& Kern, D. (1990). Appl. Phys. Lett. 56, 1841-1843.

Ade, H., Smith, A. P., Cameron, S., Cieslinski, R., Mitchell, G., Hsiao, B. \& Rightor, E. (1995). Polymer, 36, 1843-1848.

Ade, H. \& Urquhart, S. (2002). Chemical Applications of Synchrotron Radiation, edited by T. K. Sham. Singapore: World Scientific.

Ade, H., Zhang, X., Cameron, S., Costello, C., Kirz, J. \& Williams, S. (1992). Science, 258, 972.

Anderson, E. H., Olynick, D. L., Harteneck, B., Veklerov, E., Denbeaux, G., Chao, W. L., Lucero, A., Johnson, L. \& Attwood, D. (2000). J. Vac. Sci. Technol. B, 18, 2970-2975.

Botto, R. E., Cody, G. D., Kirz, J., Ade, H., Behal, S. \& Disko, M. (1994). Energy Fuels, 8, 151-154.
Buckley, C., Rarback, H., Alforque, R., Shu, D., Ade, H., Hellman, S., Iskander, N., Kirz, J., Lindaas, S., McNulty, I., Oversluizen, M., Tang, E., Attwood, D., DiGennaro, R., Howells, M., Jacobsen, C., Vladimirsky, Y., Rothman, S., Kern, D. \& Sayre, D. (1989). Rev. Sci. Instrum. 60, 2444-2447.

Cody, G. D., Ade, H., Wirick, S., Mitchell, G. D. \& Davis, A. (1998). Org. Geochem. 28, 441-455.

Coffey, T., Urquhart, S. G. \& Ade, H. (2002). J. Electron Spectrosc. Relat. Phenom. 122, 65-78.

Denbeaux, G., Anderson, E., Chao, W., Eimuller, T., Johnson, L., Kohler, M., Larabell, C., Legros, M., Fischer, P., Pearson, A., Schultz, G., Yager, D. \& Attwood, D. (2001). Nucl. Instrum. Methods A, 467/468, 841-844.

Feser, M., Carlucci-Dayton, M., Jacobsen, C., Kirz, J., Neuhäusler, U., Smith, G. \& Yu, B. (1998). Proc. SPIE, 3449, 19.

Flynn, G. J., Keller, L. P., Midler, M. A., Jacobsen, C. \& Wirick, S. (1998). Lunar and Planetary Science XXIX. Abstract \#1156.

Guttmann, P., Niemann, B., Thieme, J., Hambach, D., Schneider, G., Wiesemann, U., Rudolph, D. \& Schmahl, G. (2001). Nucl. Instrum. Methods A, 468, 849-852.

Hitchcock, A. P., Koprinarov, I., Croll, L. M., Stöver, H. \& Kneedler, E. M. (2003). In preparation.

Jacobsen, C., Kirz, J. \& Williams, S. (1992). Ultramicroscopy, 47, 55-79.

Jacobsen, C., Williams, S., Anderson, E., Brown, M. T., Buckley, C. J., Kern, D., Kirz, J., Rivers, M. \& Zhang, X. (1991). Opt. Commun. 86, 351-364.

Jacobsen, C., Wirick, S., Flynn, G. \& Zimba, C. (2000). J. Microsc. 197, 173-184.

Kaulich, B., Oestreich, S., Salome, M., Barrett, R., Susini, J., Wilhein, T., Fabrizio, E. D., Gentili, M. \& Charalambous, P. (1999). Appl. Phys. Lett. 75, 4061-4063.

Keller, L. P., Messenger, S., Flynn, G. J., Jacobsen, C. \& Wirick, S. (2000). Meteoritics Planet. Sci. 35, A86-87.

Kenney, J. M., Morrison, G. R., Browne, M. T., Buckley, C. J., Burge, R. E., Cave, R. C., Charalambous, P. S., Duke, P. J., Hare, A. R., Hills, C. P. B., Michette, A. G., Ogawa, K. \& Rogoyski, A. M. (1989). J. Phy. E Sci. Instrum. 22, 234-238.

Kim, S. K., Kortright, J. B. \& Shin, S. C. (2001). Appl. Phys. Lett. 78, 2742-2744.

Kirz, J., Ade, H., Jacobsen, C., Ko, C.-H., Lindaas, S., McNulty, I., Sayre, D., Williams, S., Zhang, X. \& Howells, M. (1992). Rev. Sci. Instrum. 63, 557563.

Kirz, J., Jacobsen, C. \& Howells, M. (1995). Q. Rev. Biophys. 28, 33-130.

Kirz, J. \& Rarback, H. (1985). Rev. Sci. Instrum. 56, 1-13.

Ko, C.-H., Kirz, J., Ade, H., Johnson, E., Hulbert, S. \& Anderson, E. (1995). Rev. Sci. Instrum. 66, 1416-1418.

Ko, C.-H., Klauser, R., Wei, D. H., Chan, H. H. \& Chuang, T. J. (1998). J. Synchrotron Rad. 5, 299-304.

McNulty, I., Frigo, S. P., Retsch, C. C., Wang, Y., Feng, Y. P., Qian, Y., Trakhtenberg, E., Tieman, B., Cha, B. C., Goetze, K., Mooney, T. \& Haddad, W. S. (1998). Proc. SPIE, 3449, 67-74.

Marsi, M., Casalis, L., Gregoratti, L., Günther, S., Kolmakov, A., Kovac, J., Lonza, D. \& Kiskinova, M. (1997). J. Electron Spectrosc. Relat. Phenom. 84, 73-83.

Maser, J., Osanna, A., Wang, Y., Jacobsen, C., Kirz, J., Spector, S., Winn, B. \& Tennant, D. (2000). J. Microsc. 197, 68-79.

Michette, A. G. (1986). Optical Systems for Soft X-rays. New York/London: Plenum Press.

Mitchell, G. E., Wilson, L. R., Dineen, M. T., Urquhart, S. G., Hayes, F., Rightor, E. G., Hitchcock, A. P. \& Ade, H. (2002). Macromolecules, 35, 1336-1341.

Neuhäusler, U., Abend, S., Jacobsen, C. \& Lagaly, G. (1999). Colloid Polym. Sci. 277, 719-726.

Ng, W., Ray-Chaudhuri, A. K., Liang, S., Singh, S., Solak, H., Welnak, J., Cerrina, F., Margaritondo, G., Underwood, J. H., Kortright, J. B. \& Perera, R. C. C. (1994). Nucl. Instrum. Methods Phys. Res. A, 347, 422.

Plaschke, M., Rothe, J., Schafer, T., Denecke, M. A., Dardenne, K., Pompe, S. \& Heize, K. H. (2002). Colloid Surface A, 197, 245-256.

Rarback, H., Kenney, J. M., Kirz, J., Howells, M. R., Chang, P., Coane, P. J., Feder, R., Jouzego, P. J., Kern D. P. \& Sayre, D. (1984). X-ray Microscopy, edited by G. Schmahl \& D. Rudolf. Berlin: Springer.

Rarback, H., Shu, D., Feng, S. C., Ade, H., Kirz, J., McNulty, L., Kern, D., Chang, T. H. P., Vladimirsky, Y., Iskander, N., Attwood, D., McQuaid, K. \& Rothman, S. (1988). Rev. Sci. Instrum. 59, 52-59.

Rightor, E. G., Hitchcock, A. P., Ade, H., Leapman, R. D., Urquhart, S. G., Smith, A. P., Mitchell, G., Fisher, D., Shin, H. J. \& Warwick, T. (1997). J. Phys. Chem., B101, 1950-1960.

Schneider, G., Schliebe, T. \& Aschoff, H. (1995). J. Vac. Sci. Technol. B, 13, 2809.

Shin, H. J. \& Lee, M. K. (2001). Nucl. Instrum. Methods Phys. Res. A, 467, 909. 
Shu, D., Siddons, D. P., Rarback, H. \& Kirz, J. (1988). Nucl. Instrum. Methods A, 266, 313-317.

Spector, S., Jacobsen, C. \& Tennant, D. (1997). J. Vac. Sci. Technol. B, 15, 2872-2876.

Stöhr, J. (1992). NEXAFS Spectroscopy. Berlin: Springer-Verlag.

Thur, W., Lauritzen, T., DeMarco, R., Baldock, B. \& Rex, K. (1997). Internal Note LBNL-40963. Lawrence Berkeley National Laboratory, Berkeley, CA, USA.

Wang, Y., Jacobsen, C., Maser, J. \& Osanna, A. (2000). J. Microsc. 197, 80-93.

Warwick, T., Ade, H., Kilcoyne, A. L. D., Kritscher, M., Tylisczcak, T., Fakra, S., Hitchcock, P., Hitchcock, A. P. \& Padmore, H. A. (2002). J. Synchrotron Rad. 9, 254-257.

Warwick, T., Franck, K., Kortright, J., Meigs, G., Moronne, M., Myneni, S., Rotenberg, E., Seal, S., Steele, W. F., Ade, H., Garcia, A., Cerasari, S., Denlinger, J., Hayakawa, S., Hitchcock, A. P., Tyliszczak, T., Kikuma, J.,
Rightor, E. G., Shin, H.-J. \& Tonner, B. P. (1998). Rev. Sci. Instrum. 69, 2964-2973.

Warwick, T., Meigs, G., Morisson, G. \& Ade, H. (1999). ALS Internal Note LSBL517. Advanced Light Source, Berkeley, CA, USA.

Welnak, J., Dong, Z., Solak, H., Wallace, J., Cerrina, F., Bertolo, M., Bianco, A., Fonzo, S. D., Fontana, S., Jark, W., Mazzolini, F., Rosei, R., Savoia, A., Underwood, J. H. \& Margaritondo, G. (1995). Rev. Sci. Instrum. 66, 2273 2276 .

Winesett, D. A. \& Ade, H. (1997). X-ray Microscopy and Spectromicroscopy, edited by J. Thieme, G. Schmahl, E. Umbach \& D. Rudolph. Berlin: Springer-Verlag.

Winn, B., Ade, H., Buckley, C., Feser, M., Howells, M., Hulbert, S., Jacobsen, C., Kaznacheyev, K., Kirz, J., Osanna, A., Maser, J., McNulty, I., Miao, J., Oversluizen, T., Spector, S., Sullivan, B., Wang, Y., Wirick, S. \& Zhang, H. (2000). J. Synchrotron Rad. 7, 395-404. 